\title{
Clinical Scenario Classification for Characterization and Outcome Prediction of Acute Decompensated Heart Failure Under Contemporary Phenotyping
}

\author{
Jin Komuro, MD; Yuji Nagatomo, MD; Keitaro Mahara, MD; Mitsuaki Isobe, MD; \\ Ayumi Goda, MD; Yasumori Sujino, MD; Atsushi Mizuno, MD; Yasuyuki Shiraishi, MD; \\ Takashi Kohno, MD; Shun Kohsaka, MD; Tsutomu Yoshikawa, MD; \\ West Tokyo Heart Failure (WET-HF) Registry Investigators
}

\begin{abstract}
Background: The concept of Clinical Scenario (CS) classification has been widely utilized to aid in choosing appropriate management strategies for acute decompensated heart failure (ADHF).

Methods and Results: The West Tokyo-Heart Failure (WET-HF) Registry is a multicenter, prospective cohort registry enrolling consecutive hospitalized ADHF patients. Based on systolic blood pressure (SBP) at admission, 4,000 patients enrolled between 2006 and 2017 were classified into 3 groups: CS1, SBP $\geq 140 \mathrm{mmHg}$; CS2, $100 \leq S B P<140 \mathrm{mmHg}$; and CS3, SBP <100 mmHg. The CS1 group had a high rate of fluid retention such as leg edema, and the largest reduction in body weight at discharge. In-hospital diuretics use was the most frequent in CS1. Although the primary endpoint of long-term all-cause death and/or ADHF re-hospitalization was more common in more advanced CS, there was no significant difference between the 3 CS groups in patients with HF with preserved ejection fraction (HFpEF; $P=0.10$ ). Although more advanced $C S$ was associated with larger left ventricular (LV) chamber size in $\mathrm{HF}$ with reduced $\mathrm{EF}$ (HFrEF), it was associated with smaller LV size in HFpEF.
\end{abstract}

Conclusions: The long-term prognostic value of CS classification was limited in HFpEF. Whereas CS was closely associated with degree of $\mathrm{LV}$ remodeling in HFrEF, a smaller LV chamber might be associated with a lower cardiovascular functional reserve in HFpEF.

Key Words: Clinical Scenario; Heart failure; Systolic blood pressure

$\mathbf{H}$ eart failure (HF) is a clinical syndrome characterized by typical symptoms that may be accompanied by signs resulting from a structural and/or functional cardiac abnormality that lead to a reduced cardiac output and/or elevated intracardiac pressure. ${ }^{1}$ Choosing the appropriate initial treatment strategy is important in achieving the optimal outcome of acute decompensated HF (ADHF). ${ }^{1,2}$ A decade ago, Mebazaa et al proposed the Clinical Scenario (CS) classification as a tool to manage patients with ADHF in the prehospital and early in-hospital phase, given the lack of large randomized, controlled, clinical trials in this area. ${ }^{3} \mathrm{CS}$ is a unique system that is based on the initial systolic blood pressure (SBP) and other symptoms, as follows: CS1, SBP $\geq 140 \mathrm{mmHg}$; CS2, $100 \leq \mathrm{SBP}<140 \mathrm{mmHg}$; CS3, SBP < 100 mmHg; CS4,
ADHF with signs of acute coronary syndrome (ACS); and CS5, isolated right ventricular failure. ${ }^{3}$ Although the concept of CS has been widely accepted, the characteristics, details of in-hospital management, and subsequent outcome of ADHF patients, by CS class, have not been reported widely. Accumulating evidence suggests that the pathology of HF differs vastly according to phenotype. In particular, $\mathrm{HF}$ with preserved ejection fraction (HFpEF) presents a substantial public health and socioeconomic burden due to its explosively increasing prevalence and limited evidence of effective treatment. HFpEF is a clinical entity that has a distinct pathophysiology from HF with reduced ejection fraction (HFrEF). ${ }^{4}$ This might lead to variation in the significance of CS, given the differing impact of SBP in HFpEF vs. HFrEF for patients with compensated status. ${ }^{5}$

Received November 20, 2018; revised manuscript received February 19, 2019; accepted February 22, 2019; J-STAGE Advance Publication released online March 28, 2019 Time for primary review: 1 day

Department of Cardiology, Sakakibara Heart Institute, Fuchu (J.K., Y.N., K.M., M.I., T.Y.); Department of Cardiology, Keio University School of Medicine, Tokyo (J.K., Y. Shiraishi, T.K., S.K.); Department of Cardiology, National Defense Medical College, Tokorozawa (Y.N.); Division of Cardiology, Kyorin University School of Medicine, Tokyo (A.G.); Department of Cardiology, Saitama Medical University, International Medical Center, Hidaka (Y. Sujino); and Department of Cardiology, St. Luke's International Hospital, Tokyo (A.M.), Japan

Mailing address: Yuji Nagatomo, MD, Department of Cardiology, National Defense Medical College, 3-2 Namiki, Tokorozawa 359-8513, Japan. E-mail: con401@ndmc.ac.jp

ISSN-2434-0790 All rights are reserved to the Japanese Circulation Society. For permissions, please e-mail: cr@j-circ.or.jp 


\begin{tabular}{|c|c|c|c|c|}
\hline & $\begin{array}{c}\text { CS1 } \\
(n=1,862)\end{array}$ & $\underset{(n=1,779)}{\text { CS2 }}$ & $\underset{(n=351)}{C S 3}$ & $P$ value \\
\hline \multicolumn{5}{|l|}{ Patient characteristics at admission } \\
\hline Age (years) & $79(68-84)$ & $77(67-84)^{\star \star, \dagger}$ & $73(61-82)^{\star \star,+, \ddagger}$ & $<0.001$ \\
\hline Male & $1,126 / 1,862(60)$ & $1,068 / 1,779(60)$ & $195 / 351(56)$ & 0.22 \\
\hline $\mathrm{BMI}\left(\mathrm{kg} / \mathrm{m}^{2}\right)$ & $23.2(20.7-26.1)$ & $22.8(20.3-25.6)^{\star \star, \dagger}$ & $21.4(18.9-24.1)^{\star *, t, \neq}$ & $<0.001$ \\
\hline Etiology & & & & $<0.001$ \\
\hline $\mathrm{DCM}$ & $194 / 1,862(10)$ & $271 / 1,779(15)$ & $83 / 351(24)$ & \\
\hline ICM & $595 / 1,862(32)$ & $481 / 1,779(27)$ & $88 / 351(25)$ & \\
\hline VHD & $451 / 1,862(24)$ & $520 / 1,779(29)$ & $77 / 351(22)$ & \\
\hline Previous HF admission & $431 / 1,846(23)$ & $590 / 1,764(33)$ & $174 / 346(50)$ & $<0.001$ \\
\hline \multicolumn{5}{|l|}{ Comorbidity } \\
\hline Cerebral infarction & $256 / 1,859(14)$ & $250 / 1,770(14)$ & $42 / 348(12)$ & 0.60 \\
\hline COPD & $97 / 1,854(5.2)$ & $87 / 1,769(4.9)$ & $15 / 350(4.3)$ & 0.74 \\
\hline Hypertension & $1,439 / 1,861(77)$ & $1,039 / 1,779(58)$ & $154 / 351(44)$ & $<0.001$ \\
\hline Dyslipidemia & $765 / 1,852(41)$ & $642 / 1,762(36)$ & $118 / 348(34)$ & 0.002 \\
\hline Diabetes mellitus & $689 / 1,860(37)$ & $572 / 1,779$ (32) & $111 / 350(32)$ & 0.004 \\
\hline Severe renal failure with Dialysis & $81 / 1,859(4.4)$ & $42 / 1,777(2.4)$ & $15 / 350(4.3)$ & 0.003 \\
\hline $\mathrm{AF}$ & $760 / 1,856(41)$ & $940 / 1,776(53)$ & $186 / 349(53)$ & $<0.001$ \\
\hline $\mathrm{SBP}(\mathrm{mmHg})$ & $161(149-181)$ & $122(111-130)^{\star *, \dagger}$ & $91(84-96)^{\star *,+, \ddagger}$ & $<0.001$ \\
\hline HR (beats/min) & $95(76-116)$ & $88(72-109)^{\star \star, \dagger}$ & $82(70-102)^{\star *,+, \ddagger}$ & $<0.001$ \\
\hline $\mathrm{SpO}_{2}(\%)$ & $95(92-98)$ & $96(93-98)^{\star *, \dagger}$ & $96(95-98)^{\star *,+, \neq}$ & $<0.001$ \\
\hline Paroxysmal nocturnal dyspnea & $706 / 1,735(41)$ & $552 / 1,664(33)$ & 95/325 (29) & $<0.001$ \\
\hline Orthopnea & $821 / 1,589(52)$ & $589 / 1,569(38)$ & $109 / 298(37)$ & $<0.001$ \\
\hline Crackles & $1,027 / 1,738(59)$ & $766 / 1,675(46)$ & 136/327 (42) & $<0.001$ \\
\hline Third sound & $665 / 1,748(38)$ & $620 / 1,700(36)$ & $114 / 330(35)$ & 0.39 \\
\hline Jugular vein engorgement & $759 / 1,603(47)$ & $740 / 1,582(47)$ & $125 / 298(42)$ & 0.23 \\
\hline Lower leg edema & $1,223 / 1,788(68)$ & $1,169 / 1,720(68)$ & $182 / 329(55)$ & $<0.001$ \\
\hline Cold extremity & $357 / 1,538(23)$ & $273 / 1,510(18)$ & 90/294 (31) & $<0.001$ \\
\hline NYHA (II/III/IV) & $321 / 577 / 871(18 / 33 / 49)$ & $344 / 723 / 618(20 / 43 / 37)$ & $58 / 121 / 143(18 / 38 / 44)$ & $<0.001$ \\
\hline BNP (pg/mL) & $755(400.4-1,351.6)$ & $668.7(331.3-1,157.1)^{\star \star, \dagger}$ & $763.6(416.7-1,391.9)$ & 0.002 \\
\hline eGFR $\left(\mathrm{mL} / \mathrm{min} / 1.73 \mathrm{~m}^{2}\right)$ & $49.8(33.3-64.5)$ & $49.3(34.2-64.6)$ & $42.6(26.5-60.1)^{\star *,+, \neq}$ & $<0.001$ \\
\hline $\mathrm{Hb}(\mathrm{g} / \mathrm{dL})$ & $12.0(10.3-13.7)$ & $12.0(10.4-13.7)$ & $11.9(10.1-13.4)$ & 0.26 \\
\hline $\mathrm{Na}(\mathrm{mEq} / \mathrm{L})$ & $140(138-142)$ & $140(137-142)^{\star *, \dagger}$ & $138(135-141)^{\star *, t, \neq}$ & $<0.001$ \\
\hline LVDd (mm) & $51(45-57)$ & $52(45-59)^{\star \star, \dagger}$ & $56(45-64)^{\star \star, \dagger, *, \neq}$ & $<0.001$ \\
\hline LVDs (mm) & $38(31-47)$ & $39(31-51)^{* *, \dagger}$ & $44(32-56)^{* *,+, \neq}$ & $<0.001$ \\
\hline LAD (mm) & $43(38-48)$ & $45(40-50)^{* *, \dagger}$ & $44(39-50)$ & $<0.001$ \\
\hline LVEF (\%) & $47(35-59)$ & $44(30-58)^{* *, \dagger}$ & $36(24-53)^{* *,+, \neq}$ & $<0.001$ \\
\hline \multicolumn{5}{|l|}{ In-hospital variables } \\
\hline Length of stay & $14(9-22)$ & $15(10-24)^{*, \dagger}$ & $18(11-33)^{* *,+, \neq}$ & $<0.001$ \\
\hline In-hospital mortality & $47 / 1,862(2.5)$ & $76 / 1,779(4.3)$ & $41 / 351(11.7)$ & $<0.001$ \\
\hline \multicolumn{5}{|l|}{ Patient characteristics at discharge } \\
\hline $\mathrm{SBP}(\mathrm{mmHg})$ & $118(106-130)$ & $106(98-118)^{\star *, \dagger}$ & $98(88-108)^{\star *,+, \neq}$ & $<0.001$ \\
\hline HR (beats/min) & $70(61-78)$ & $70(62-80)^{\star \star, \dagger}$ & $71(62-80)$ & 0.004 \\
\hline$\Delta \mathrm{BW}(\mathrm{kg})$ & $-3.8(-6.4$ to -1.6$)$ & $-3.6(-6.3$ to -1.5$)$ & $-2.4(-5.6 \text { to }-0.6)^{\star \star, t, t}$ & $<0.001$ \\
\hline
\end{tabular}

Data given as $\mathrm{n}(\%)$ or median (IQR). ${ }^{*} \mathrm{P}<0.05$, ${ }^{* *} \mathrm{P}<0.01$ tVs. $\mathrm{CS} 1$; $\neq$ vs. CS2. $\Delta \mathrm{BW}$, difference in body weight between admission and discharge. ACEI, angiotensin converting enzyme inhibitor; ADHF, acute decompensated heart failure; AF, atrial fibrillation; ARB, angiotensin receptor blocker; BMI, body mass index; BNP, B-type natriuretic peptide; CS, Clinical Scenario; DCM, dilated cardiomyopathy; eGFR, estimated glomerular filtration rate; Hb, hemoglobin; HF, heart failure; HR, heart rate; ICM, ischemic cardiomyopathy; LAD, left atrial dimension; LVDd, left ventricular end-diastolic diameter; LVDs, left ventricular end-systolic diameter; LVEF, left ventricular ejection fraction; MRA, mineralocorticoid receptor antagonist; $\mathrm{Na}$, serum sodium; $\mathrm{SBP}$, systolic blood pressure; $\mathrm{SpO}_{2}$, saturation of peripheral oxygen; VHD, valvular heart disease.

In the present study, we sought to validate the real-world clinical use of CS and to determine its value for the characterization of patients with ADHF, including the short-term and long-term prognosis, in the multicenter West Tokyo-Heart Failure (WET-HF) registry. Further, we sought to evaluate the significance of CS according to phenotype of HF: that is, HFrEF or HFpEF.

\section{Methods}

\section{Study Design}

The WET-HF Registry is an ongoing, prospective, multicenter cohort registry designed to collect data on the clinical background and outcome of patients hospitalized for ADHF ${ }^{6,7}$ A total of 4,000 consecutive patients who were 


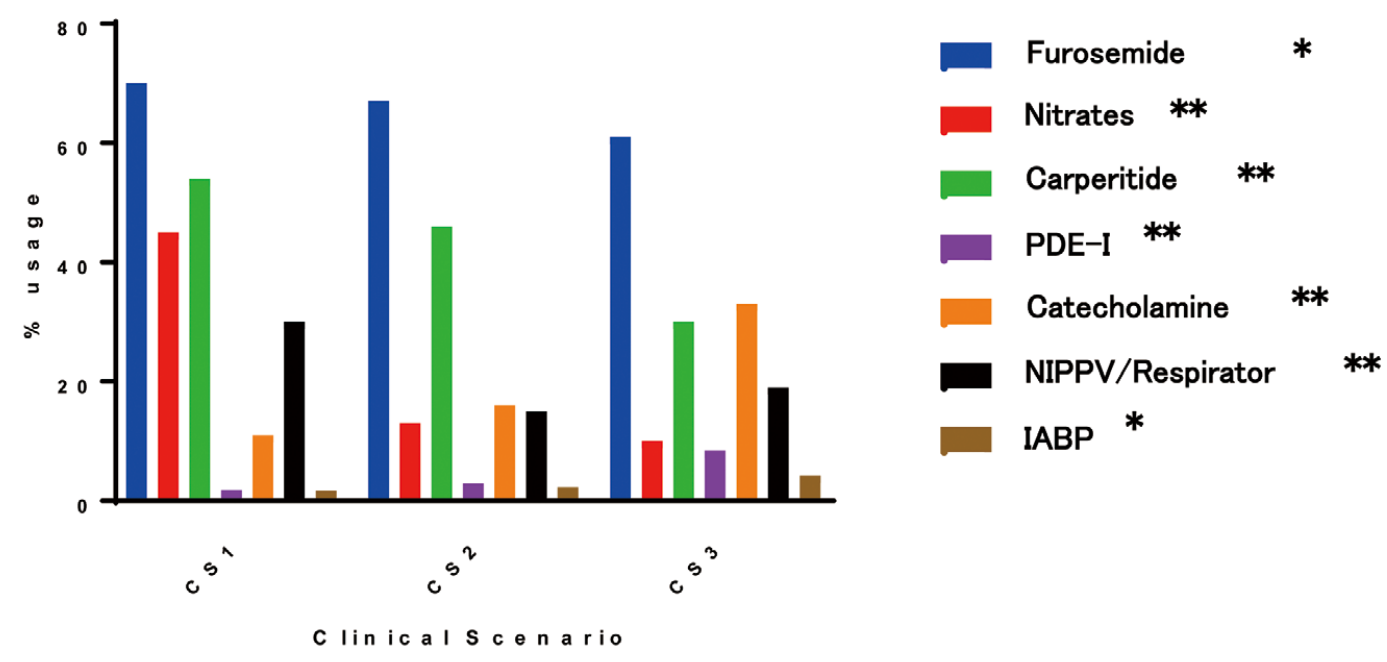

Figure 1. In-hospital treatment in acute decompensated heart failure patients according to Clinical Scenario (CS) group. IABP, intra-aortic balloon pump; NIPPV, non-invasive positive pressure ventilation; PDEl, phosphodiesterase-III inhibitor. ${ }^{\star} \mathrm{P}<0.05$, ${ }^{\star *} \mathrm{P}<0.001$ between CS1-3.

admitted to hospitals participating in the multicenter WET-HF Registry for ADHF between January 2006 and December 2017 were enrolled. Data are collected by trained personnel from the patient charts and entered using a Web-based electronic data capture system. ADHF was defined as rapid-onset HF or a change in the signs and symptoms of HF requiring urgent therapy and hospitalization, based on the Framingham criteria. ${ }^{8}$ Patients presenting with ACS or isolated right-sided HF were excluded. The clinical diagnosis of ADHF was made by the individual cardiologists at each institution. Exclusive on-site auditing by the investigators (Y. Shiraishi and S.K.) ensured proper registration of each patient. Before the launch of the WETHF registry, information on the objective of the present study and its social significance and an abstract were provided for clinical trial registration with the University Hospital Medical Information Network (UMIN000001171). The study protocol was approved by the institutional review boards at each site, and research was conducted in accordance with the Declaration of Helsinki. Written and/ or oral informed consent were obtained from each subject before the study.

\section{Subjects}

After excluding 8 cases of missing SBP data at admission $(1.0 \%)$, a total of 3,992 subjects were available for analysis. Based on SBP at admission, the subjects were classified into 3 groups: CS1, SBP $\geq 140 \mathrm{mmHg}, \mathrm{n}=1,862(46.6 \%)$; $\mathrm{CS} 2,100 \leq \mathrm{SBP}<140 \mathrm{mmHg}, \mathrm{n}=1,779(44.6 \%)$; and $\mathrm{CS} 3$, SBP $<100 \mathrm{mmHg}, \mathrm{n}=351(8.8 \%)$.

In-hospital treatment for ADHF such as i.v. agents and non-pharmacological therapy was recorded. Carperitide is an i.v. recombinant atrial natriuretic peptide approved only in Japan for ADHF, whereas nesiritide (a recombinant B-type natriuretic peptide) is available in USA, Argentina, Columbia, Switzerland, and Israel. ${ }^{9}$ The expected cardiovascular effects of carperitide are vasodilation and sympathetic tone reduction in the peripheral vasculature. ${ }^{10}$

\section{Outcomes}

We collected the data on in-hospital mortality as a shortterm outcome measure. The subjects were also followed up after discharge for the primary endpoint, defined as allcause death or re-hospitalization for ADHF in $\leq 1,000$ days as a long-term outcome measure.

\section{Statistical Analysis}

In this study, we defined HFpEF as HF with left ventricular ejection fraction (LVEF) $\geq 50 \%, \mathrm{HFrEF}$ as $\mathrm{HF}$ with LVEF $<40 \%$, and $\mathrm{HF}$ with mid-range ejection fraction (HFmrEF) as HF with LVEF 40-49\%.1 Continuous variables are presented as median (IQR). Kruskal-Wallis test was used to evaluate the differences between the $3 \mathrm{CS}$ groups, and when it was significant, Steel-Dwass test was additionally done to compare each CS group. To explore the effect of CS or CS-guided in-hospital treatment on in-hospital mortality as a short-term outcome, multivariate logistic regression analysis was performed for the overall population and each CS subgroup adjusting for covariates such as age, gender, LVEF, hemoglobin ( $\mathrm{Hb})$, estimated glomerular filtration rate (eGFR), and total bilirubin (TB). For survival analysis, Kaplan-Meier curves and log-rank test were used to assess the statistical difference in eventfree survival from primary endpoint or re-hospitalization for ADHF between the $3 \mathrm{CS}$ groups. Cox proportional hazard model analysis was performed to determine the independent predictors of primary endpoint or re-hospitalization for ADHF. To test the prognostic significance of CS, other potential confounding factors, including inhospital treatment, were used as covariates. Multiple regression analysis was performed to identify the factors related to $\mathrm{CS}$. Covariates such as age, gender, body mass index (BMI), LVEF, Hb, eGFR, TB, and LV end-diastolic diameter (LVDd) were included as independent variables in this model. All statistical analysis was performed with IBM SPSS Statistics, version 24 (Armonk, NY, USA). 


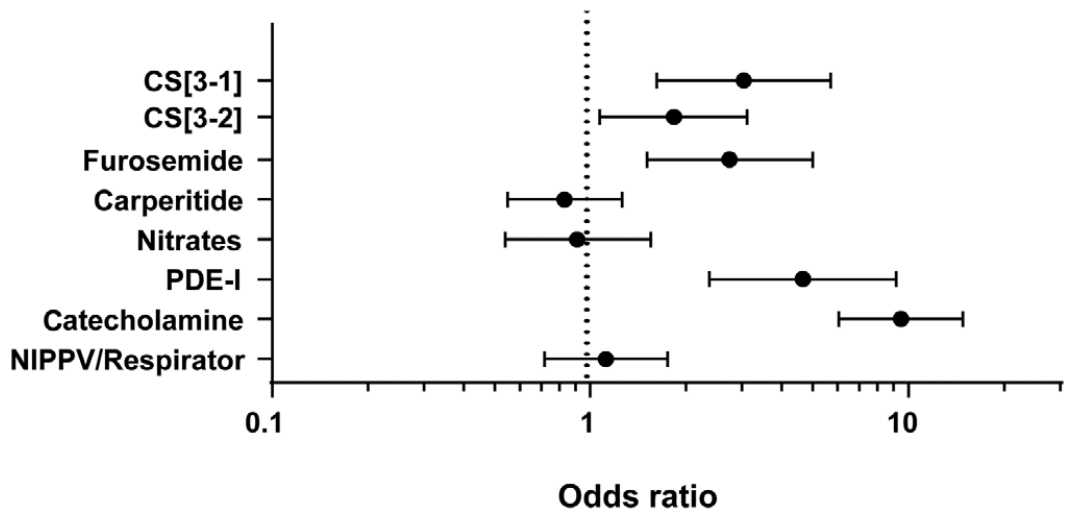

Figure 2. Multiple logistic regression analysis for in-hospital mortality in acute decompensated heart failure patients, adjusted for covariates such as age, gender, left ventricular ejection fraction, hemoglobin, estimated glomerular filtration rate and total bilirubin. CS was independently associated with higher in-hospital mortality. Abbreviations as in Figure 1.

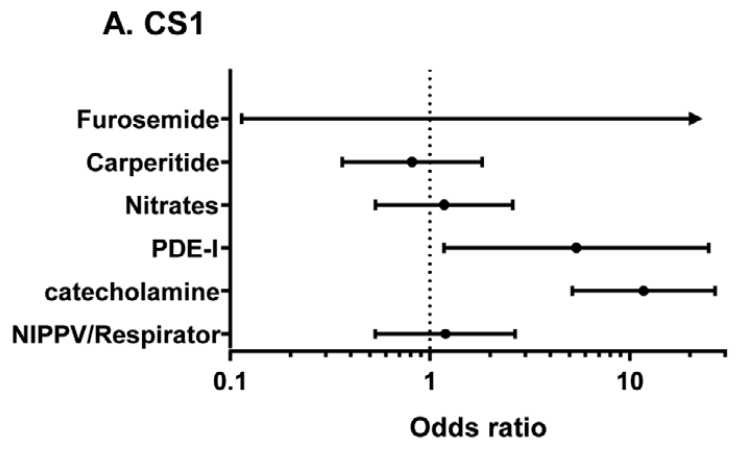

B. CS2

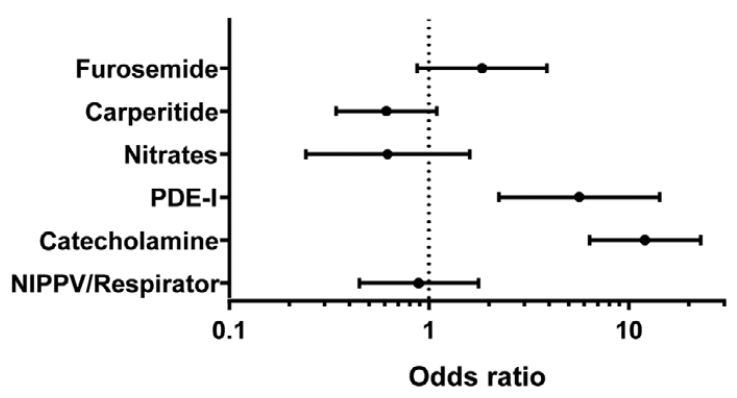

C. $\operatorname{cs3}$

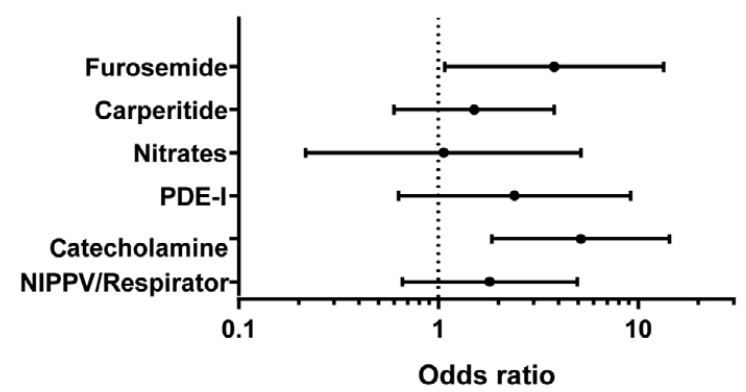

Figure 3. Multiple logistic regression analysis for in-hospital mortality in acute decompensated heart failure patients according to (A) CS1, (B) CS2 and (C) CS3 group. The odds ratio was adjusted for age, gender, left ventricular ejection fraction, hemoglobin, estimated glomerular filtration rate and total bilirubin. Abbreviations as in Figure 1.

\section{Results}

CS: Baseline Characteristics and In-Hospital Treatment

The subject baseline characteristics are listed in Table 1. At baseline, age was younger, although de novo ADHF was less common, in more advanced CS. Heart rate (HR) at admission was lower in more advanced CS. The findings related to pulmonary congestion, such as paroxysmal nocturnal dyspnea, orthopnea, and crackles, and that related to fluid retention such as leg edema were less common in more advanced CS. Conversely, cold extremities were more common in more advanced CS. LVEF was lower, and LVDd and LV end-systolic diameter (LVDs) were larger in more advanced CS.

The in-hospital treatment in each CS group is shown in Figure 1. The use of loop diuretics, nitrates, carperitide, and respirator including non-invasive positive pressure ventilation were more common, and the use of inotropes/ vasopressors and intra-aortic balloon pump (IABP) was less common in CS1 (CS1/2/3: furosemide, 69/67/62\%, $\mathrm{P}=0.0305$; nitrates, $44 / 13 / 9 \%, \mathrm{P}<0.001$; carperitide, $54 / 46 / 30 \%, \mathrm{P}<0.001$; respirator, $27 / 13 / 15 \%, \mathrm{P}<0.001$; phosphodiesterase-III inhibitor (PDEI), $2 / 3 / 8 \%, \mathrm{P}<0.001$; catecholamine, $11 / 16 / 34 \%, \mathrm{P}<0.001$; IABP, $2 / 2 / 4 \%, \mathrm{P}=0.02$, 

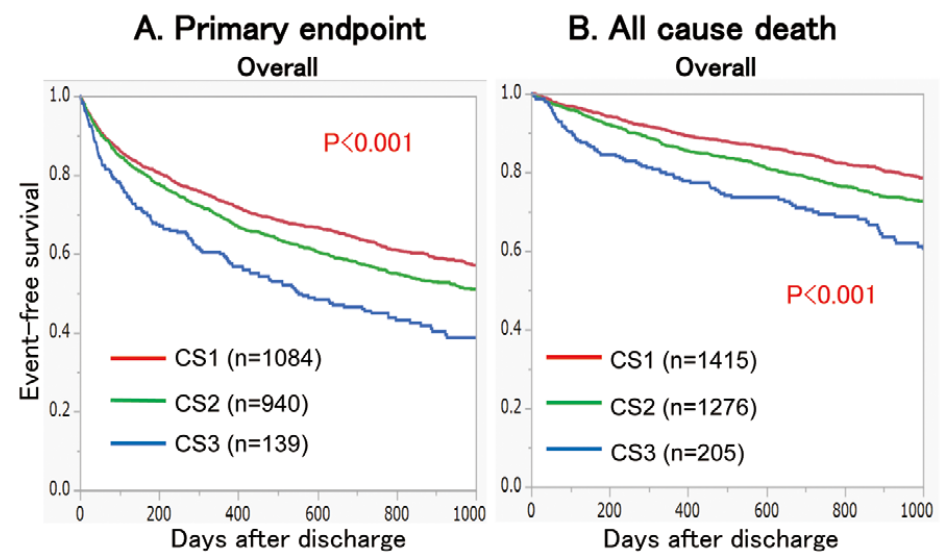

C. ADHF re-hospitalization

Overall

Figure 4. Kaplan-Meier curves for (A) primary endpoint (composite of all-cause death or re-hospitalization due to acute decompensated heart failure [ADHF]), (B) all-cause death, and (C) re-hospitalization for ADHF according to Clinical Scenario (CS) group.

\section{Primary endpoint}

A.

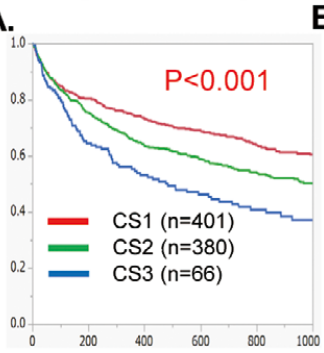

D.

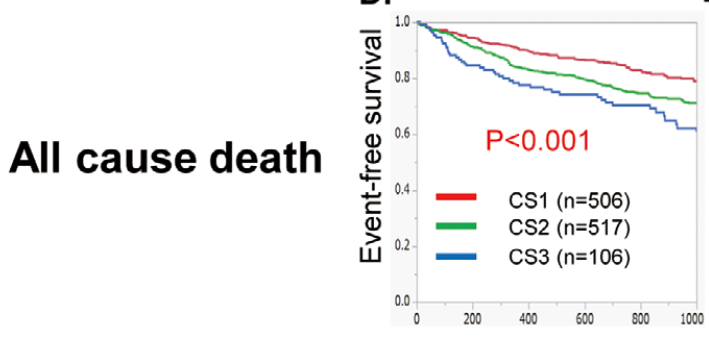

G.

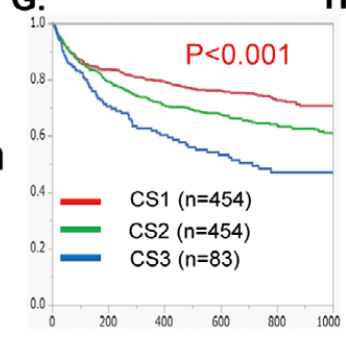

HFmrEF

B.

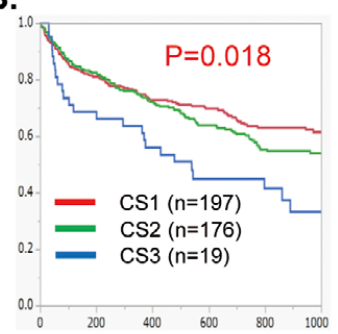

E.

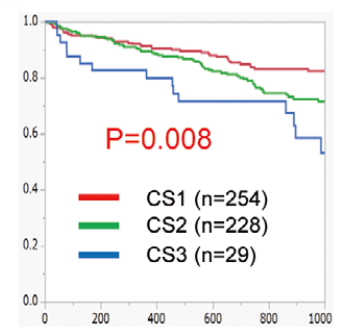

H.

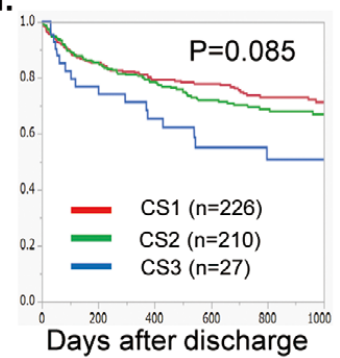

HFpEF

$(E F \geq 50)$

C.

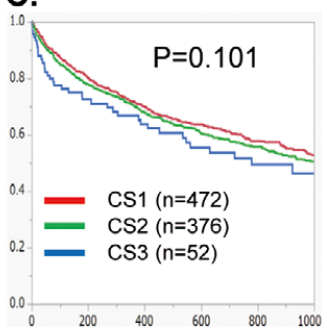

F.

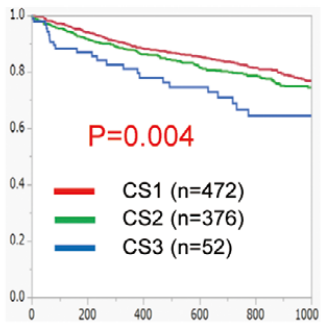

I.

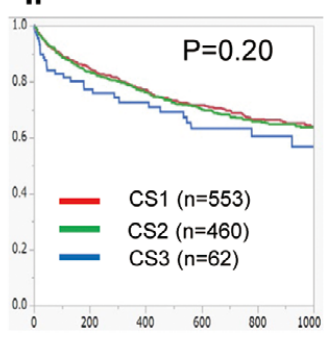

Figure 5. Kaplan-Meier curves for (A-C) primary endpoint (all cause death and re-hospitalization due to ADHF), (D-F) all-cause death, and $(\mathbf{G}-\mathbf{I})$ re-hospitalization for ADHF, in patients with $(\mathbf{A}, \mathbf{D}, \mathbf{G})$ heart failure with reduced ejection fraction (HFrEF; LVEF $<40 \%$ ); (B,E,H) heart failure with mid-range ejection fraction (HFmrEF; LVEF 40-49\%); and (C,F,I) heart failure with preserved ejection fraction (HFpEF; LVEF $\geq 50 \%$ ), according to CS group. Abbreviations as in Figure 4. 


\begin{tabular}{lccr}
\hline \multirow{2}{*}{ Table 2. Multivariate Indicators of Primary Endpoint ${ }^{+}$in ADHF Patients } & Overall \\
\cline { 2 - 4 } & \multicolumn{1}{c|}{ HR } & $95 \%$ Cl & P-value \\
Age & 1.025 & $1.019-1.031$ & $<0.001$ \\
Gender (female) & 1.011 & $0.897-1.138$ & 0.861 \\
BMI & 0.974 & $0.959-0.989$ & $<0.001$ \\
AF & 1.168 & $1.040-1.312$ & 0.009 \\
CS & & & $<0.001$ \\
(3-1) & 1.614 & $1.312-1.973$ & $<0.001$ \\
(3-2) & 1.429 & $1.177-1.724$ & $<0.001$ \\
Hb & 0.913 & $0.887-0.940$ & $<0.001$ \\
eGFR & 0.990 & $0.987-0.993$ & $<0.001$ \\
LVEF & 0.988 & $0.984-0.992$ & $<0.001$ \\
Furosemide (i.v.) & 1.060 & $0.940-1.198$ & 0.343 \\
Nitrates & 0.855 & $0.735-0.993$ & 0.042 \\
PDEI & 1.395 & $0.991-1.964$ & 0.056 \\
Catecholamine & 1.035 & $0.870-1.230$ & 0.698 \\
NIPPV/Respirator & 1.098 & $0.945-1.276$ & 0.224 \\
\hline
\end{tabular}

${ }^{\dagger}$ Composite of all-cause death or re-hospitalization due to ADHF. NIPPV, non-invasive positive pressure ventilation; PDEI, phosphodiesterase-III inhibitor. Other abbreviations as in Table 1.

Figure 1). The use of percutaneous cardiopulmonary support was similar between the $3 \mathrm{CS}$ groups $(\mathrm{CS} 1 / 2 / 3$ : $0.5 / 0.5 / 0.3 \%, \mathrm{P}=0.864)$.

\section{CS and In-Hospital/Long-Term Outcome}

Crude in-hospital mortality was higher in more advanced CS (Table 1). On logistic regression analysis CS3 was independently associated with higher in-hospital mortality after adjustment for covariates, including in-hospital treatment strategies (CS3-1: OR, 2.98; 95\% CI: 1.62-5.69, $\mathrm{P}<0.001$; CS3-2: OR, 1.83 ; 95\% CI: $1.07-3.11, \mathrm{P}=0.03$; Figure 2). Although catecholamine use was associated with higher in-hospital mortality in all CS groups, i.v. loop diuretics were associated with in-hospital death only in CS3. (Figure 3). The reduction of body weight $(\Delta \mathrm{BW})$ at discharge was the largest in CS1 (Table 1). After discharge, the primary endpoint of all-cause death or re-hospitalization for ADHF was more common in CS3 compared with CS1 and CS2 ( $\mathrm{P}<0.001, \log$-rank test, Figure 4A). Both all-cause death and HF re-hospitalization were also more common in CS3 (all-cause death, $\mathrm{P}<0.001$, Figure 4B; HF re-hospitalization, $\mathrm{P}<0.001$, Figure $4 \mathrm{C}$ ).

\section{CS in HF According to EF Status}

Although the occurrence of the primary endpoint was more common in more advanced CS in the HFrEF and HFmrEF subgroups, there was no significant difference between the CS groups in HFpEF (HFrEF, $\mathrm{P}<0.001$; HFmrEF, $\mathrm{P}=0.018$; HFpEF, $\mathrm{P}=0.101$, Figure 5A-C). All-cause death was more common in more advanced CS in all of the 3 subgroups (Figure 5D-F). Although rehospitalization for ADHF was also more common in more advanced CS in HFrEF, this was not significantly different in HFmrEF or HFpEF (HFrEF, $\mathrm{P}<0.001$; HFmrEF, $\mathrm{P}=0.085$; HFpEF, $\mathrm{P}=0.20$, Figure 5G-I). On Cox proportional hazard model analysis, CS was an independent predictor of primary endpoint, even after adjusting for covariates, including in-hospital treatment (Table 2). CS was an independent predictor of primary endpoint in HFrEF $(\mathrm{P}=0.037)$ and HFmrEF $(\mathrm{P}=0.033)$, but it was not significant in $\mathrm{HFpEF}(\mathrm{P}=0.074$, Supplementary Table).

\section{Factors Related to CS}

Although CS aids in navigating the initial treatment strategies, the mechanisms underlying the differentiation of the CS categories remain unknown. Therefore, we sought to determine the factors associated with CS. On multivariate analysis age, BMI, TB, and LVEF were associated with CS (Figure 6A). Lower LVEF was associated with more advanced CS in HFrEF, but not in HFmrEF or HFpEF (Figure 6B-D). Larger LVDd was associated with more advanced CS in HFrEF. Conversely, however, smaller LVDd was associated with more advanced CS in HFpEF (Figure 6B,D). Neither LVEF nor LVDd was associated with CS in HFmrEF (Figure 6C).

\section{Discussion}

Since Mebazaa et al proposed the CS classification a decade ago, this system has been widely utilized in actual clinical settings such as emergency rooms and intensive care units. Despite this, to date there have been no studies that have validated its clinical use in the real world. In the present study, we reported 4 main fin dings. First, the ADHF patients in the $3 \mathrm{CS}$ groups had substantially different clinical presentations, including baseline characteristics and in-hospital treatment strategies. For example, whereas CS1 was characterized by reduced HF severity (e.g., higher LVEF), and the physical fin dings related to pulmonary congestion, CS3 was characterized by more severe disease and frequent cold extremities representing low output, in line with previous reports. ${ }^{11-14}$ This suggests that CS is likely to be associated with the HF phenotype and cardiovascular reserve and to represent the primary pathophysiologic problems of each patient. Of note, unlike the initial proposal by Mebazaa et al, ${ }^{3} \mathrm{CS} 1$ was the most closely associated with flu id retention such as leg edema and $\triangle \mathrm{BW}$, assuming flu id excretion after ADHF treatment. Second, CS predicted both in-hospital outcome and future clinical adverse events, even after adjustment for 
A. Overall

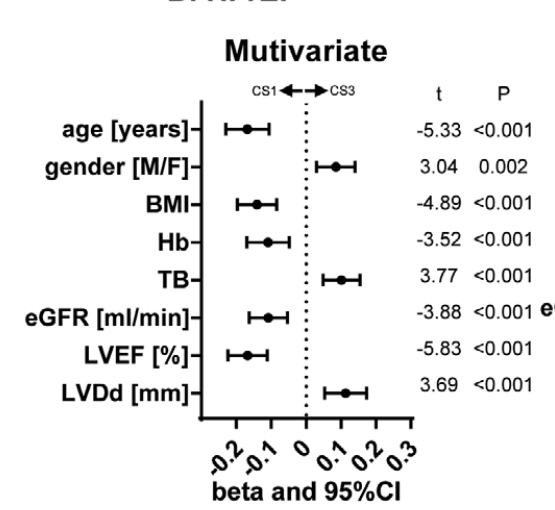

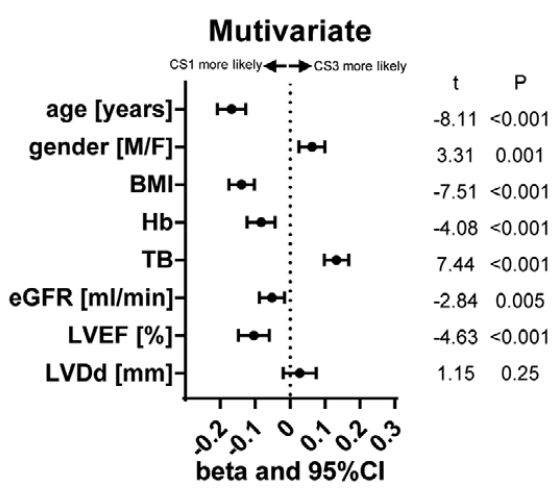

C. HFmrEF

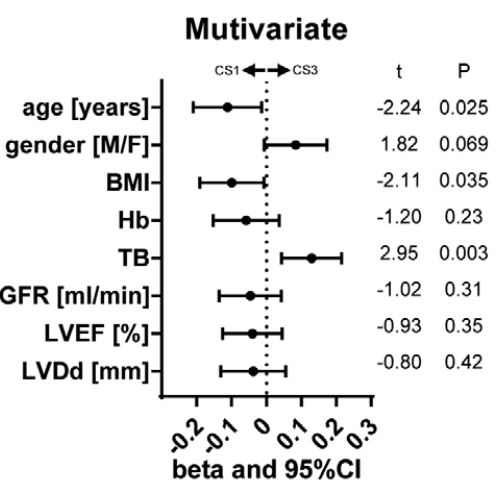

D. HFpEF

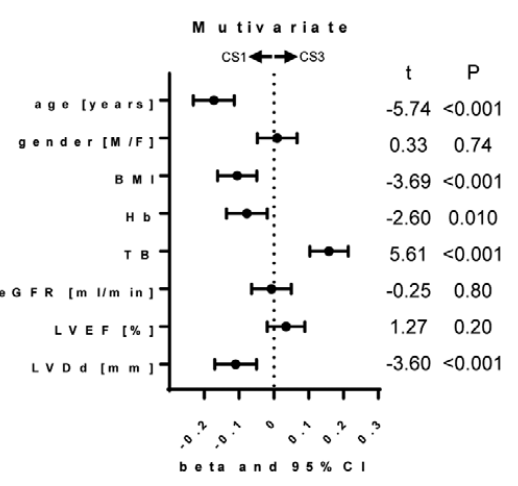

Figure 6. Multiple regression analysis for $\mathrm{CS}$ in $(\mathbf{A})$ the overall ADHF patient group; (B) the HFrEF subgroup; (C) the HFmrEF subgroup; and (D) the HFpEF subgroup. Age, gender, body mass index (BMI), hemoglobin (Hb), total bilirubin (TB), estimated glomerular filtration rate (eGFR), left ventricular ejection fraction (LVEF) and left ventricular end-diastolic diameter (LVDd) were included as independent variables in this model. Abbreviations as in Figures 4,5.

CS-guided in-hospital treatment strategies that can potentially affect them. ${ }^{\mathbf{8} 15,16}$ Third, the prognostic importance of CS was less significant in HFpEF. Fourth, CS was related to various factors, including age, BMI, and LVEF. In $\mathrm{HFrEF}, \mathrm{CS}$ was associated with measures of LV remodeling, such as LVEF and LV chamber size. On the basis of these fin dings, we concluded that CS was both a helpful measure to guide the early in-hospital management strategy and also a predictor of short-term and long-term clinical outcome. Although some HF risk scores such as the Seattle Heart Failure Mode ${ }^{17}$ have been developed and are helpful to predict long-term outcome, CS is a simpler predictor suitable for prompt evaluation of patients with ADHF. To the best of our knowledge, this is the first study to validate the clinical use of CS in the real world and its value for the characterization of patients with ADHF.

\section{Flu id Retention and Diuretic Use in CS1}

The practical recommendations by Mebazaa et al stated that minimal systemic edema is present in $\mathrm{CS}^{3}$ based on the data from the OPTIMIZE-HF registry, ${ }^{11}$ and they designated it as "vascular failure", representing inappropriate vascular constriction. In the present study, however, the higher percentage of leg edema and diuretic use was noted in $\mathrm{CS} 1$; and $\triangle \mathrm{BW}$, assuming flu id excretion after ADHF treatment, was also the largest in CS1 (Table 1). CS1 includes older patients (Table 1; Figure 1), in line with the fin dings in another Japanese HF cohort. ${ }^{18}$ It might be, at least partly, explained by the distinct dietary pattern of high salt intake in Japanese people, especially in the elderly, ${ }^{19}$ leading to retention of sodium and volume overload. ${ }^{20}$ Given that increased LV wall stiffness inevitably accompanies arterial stiffening according to LV-arterial coupling, ${ }^{21}$ in such cases volume overload can easily lead to elevated blood pressure via elevated LV end-diastolic pressure. From these fin dings, CS1 might be more associated with flu id retention representing increased preload and elevated LV end-diastolic pressure than initially proposed. Of note, the impact of diuretic use on in-hospital death was not significant in CS1, although it was in CS3 (Figure 3). Therefore, for patients in CS1, diuretics, which have no negative impact on in-hospital outcome (Figure 3A), should be used appropriately according to the degree of volume retention, contrary to the recommendation by Mebazaa et al. Diuretics, however, might have a low priority as initial treatment because a nitrate-predominant strategy was shown to be superior to a diuretic-predominant one in terms of improvement of oxygen desaturation and subsequent need for mechanical ventilation. ${ }^{22}$

\section{Inotropes in CS}

CS3 was characterized by more severe disease and frequent cold extremities representing low output. Further, the negative impact of catecholamines and PDEI on short-term 
outcome was less significant in more advanced CS (Figure 3). In particular, PDEI did not lead to increased mortality in CS3 (Figure 3C). These findings might support the recommendation from Mebazaa et al that inotropes can be used in some patients mainly in CS3, although we could not precisely examine the appropriateness of each treatment strategy because of the observational nature of the study.

\section{Chamber Size as a CS-Related Factor in HFrEF and HFpEF}

Given that CS was found to predict the future clinical outcome in subjects with ADHF, it is intriguing to explore what factors are related to CS. In the present study, multiple regression analysis indicated that various factors, including age, BMI, and LVEF, were related to CS. In HFrEF patients, lower LVEF and larger LV dimension, which precisely represent the disease process of LV remodeling in $\mathrm{HFrEF},{ }^{23}$ were associated with advanced CS. Conversely, in HFpEF patients, smaller LV size was associated with advanced CS. In HFpEF, unlike HFrEF, the time course change and the significance of LV chamber size in the disease process are controversial and not well established. ${ }^{2-26} \mathrm{~A}$ smaller LV chamber might be associated with a lower cardiovascular functional reserve in HFpEF possibly through impaired LV diastolic function, given that a smaller LV chamber might be associated with lower stroke volume ${ }^{29}$ or exercise intolerance ${ }^{30}$ in patients with HFpEF.

\section{Significance of CS in HFrEF and HFpEF}

LV remodeling in HFrEF is characterized by depressed LV systolic function and LV chamber dilatation. ${ }^{23,29}$ A more remodeled LV generally represents an advanced disease process. In the present study, in HFrEF patients, the LV diameter was larger and LVEF was lower in more advanced CS, which suggests that CS might be correlated with advanced LV remodeling: in other words, with the severity of HF. Moreover, CS was an independent predictor of primary endpoint in this subgroup. CS did, however, fail to predict outcome in $\mathrm{HFpEF}$. The mechanism underlying the differing prognostic impact of CS remains unknown. We initially speculated that it might be derived from the higher proportion of valvular heart disease (VHD) in HFpEF. Although the analysis was conducted after excluding patients with VHD, again CS failed to predict the primary endpoint in HFpEF (data not shown). As noted, the cardiac remodeling process in $\mathrm{HFpEF}$ is still obscure. ${ }^{24-26}$ Also, it has been proven that extra-cardiac burden is more significant in HFpEF than HFrEF. 30,31 Given that the patients were older, and that comorbidities such as atrial fibrillation (AF) and anemia were more common in patients with HFpEF in line with previous studies, ${ }^{30,31}$ these additional factors might also substantially affect the long-term clinical outcome. These issues might lead to the less significant prognostic value of CS in HFpEF.

\section{Study Limitations}

There are several limitations in this study. First, although we saw a difference in the in-hospital treatment strategies between the $3 \mathrm{CS}$ categories, we could not examine their appropriateness for each patient because it was an observational study. Second, although CS was originally intended to create a guide for early management strategy for
ADHF, we do not have data related to the time course of in-hospital treatment (e.g., first- and second-line therapy). Third, in the subgroup analysis, the number of patients was relatively small for comparison of the 3 CS groups, especially in the HFmrEF group. Fourth, most participants in the present study were Japanese. Caution is thus needed when applying these results to ADHF patients in other regions. Fifth, although we sought to characterize ADHF patients by CS, patients with ACS (CS4) or isolated right-sided HF (CS5) were excluded. Sixth, given that this registry was organized to elucidate the characteristics and prognosis of ADHF patients from a different point of view and it does not solely focus on the blood pressure, the method of blood pressure measurement was not unified in this study.

\section{Conclusions}

CS was simple and useful to predict short-term and longterm clinical outcome in patients with ADHF. In HFrEF, CS was closely associated with LV remodeling, and it predicted in-hospital mortality and future clinical adverse events. Although, in $\mathrm{HFpEF}$, its prognostic value was limited in contemporary clinical practice, those patients with a small LV chamber might need to be managed carefully. Further investigation is needed to elucidate the mechanism of the differing prognostic impact of CS on clinical outcome between HFrEF and HFpEF.

\section{Funding}

This study was supported by a Grant-in-Aid for Scientific Research (C) $(23591062,26461088,16$ K09469), a Health Labour Sciences Research Grant (14528506), and the Sakakibara Clinical Research Grant for the Promotion of Sciences, 2012-2017.

\section{Disclosures}

The authors declare no conflicts of interest.

\section{References}

1. Ponikowski P, Voors AA, Anker SD, Bueno H, Cleland JGF, Coats AJS, et al. 2016 ESC Guidelines for the diagnosis and treatment of acute and chronic heart failure: The Task Force for the diagnosis and treatment of acute and chronic heart failure of the European Society of Cardiology (ESC). Developed with the special contribution of the Heart Failure association (HFA) of the ESC. Eur Heart J 2016; 37: 2129-2200.

2. Goto T, Wakami K, Mori K, Kikuchi S, Fukuta H, Ohte N. Vascular physiology according to clinical scenario in patients with acute heart failure: Evaluation using the cardio-ankle vascular index. Tohoku J Exp Med 2016; 240: 57-65.

3. Mebazaa A, Gheorghiade M, Piña IL, Harjola VP, Hollenberg SM, Follath F, et al. Practical recommendations for prehospital and early in-hospital management of patients presenting with acute heart failure syndromes. Crit Care Med 2008; 36: S129S139.

4. Schwartzenberg S, Redfield MM, From AM, Sorajja P, Nishimura RA, Borlaug BA. Effects of vasodilation in heart failure with preserved or reduced ejection fraction: Implications of distinct pathophysiologies on response to therapy. J Am Coll Cardiol 2012; 59: 442-451.

5. Maeder MT, Kaye DM. Differential impact of heart rate and blood pressure on outcome in patients with heart failure with reduced versus preserved left ventricular ejection fraction. Int $J$ Cardiol 2012; 155: 249-256.

6. Shiraishi Y, Sawano M, Kohno T, Nishiyama T, Maekawa Y, Sano M, et al. Validation of the Seattle heart failure model in Japanese heart failure patients. Int J Cardiol 2016; 203: 87-89.

7. Yagawa M, Nagatomo Y, Izumi Y, Mahara K, Tomoike H, Shiraishi Y, et al. Effect of obesity on the prognostic impact of atrial fibrillation in heart failure with preserved ejection fraction. Circ J 2017; 81: 966-973. 
8. Lefkowitz RJ, Rockman HA, Koch WJ. Catecholamines, cardiac $\beta$-adrenergic receptors, and heart failure. Circulation 2000; 101: 1634-1637.

9. Konishi M, Ishida J, Springer J, von Haehling S, Akashi YJ, Shimokawa $\mathrm{H}$, et al. Heart failure epidemiology and novel treatments in Japan: Facts and numbers. ESC Heart Fail 2016; 3: $145-151$.

10. Marin-Grez M, Fleming JT, Steinhausen M. Atrial natriuretic peptide causes pre-glomerular vasodilatation and post-glomerular vasoconstriction in rat kidney. Nature 1986; 324: 473 .

11. Gheorghiade M, Abraham WT, Albert NM, Greenberg BH, O'Connor CM, She L, et al. Systolic blood pressure at admission, clinical characteristics, and outcomes in patients hospitalized with acute heart failure. JAMA 2006; 296: 2217-2226.

12. Buiciuc O, Rusinaru D, Lévy F, Peltier M, Slama M, Leborgne $\mathrm{L}$, et al. Low systolic blood pressure at admission predicts longterm mortality in heart failure with preserved ejection fraction. $J$ Card Fail 2011; 17: 907-915.

13. Rosman Y, Kopel E, Shlomai G, Goldenberg I, Grossman E. The association between admission systolic blood pressure of heart failure patients with preserved systolic function and mortality outcomes. Eur J Intern Med 2015; 26: 807-812.

14. Al-Lawati JA, Sulaiman KJ, Al-Zakwani I, Alsheikh-Ali AA, Panduranga P, Al-Habib KF, et al. Systolic blood pressure on admission and mortality in patients hospitalized with acute heart failure: Observations from the Gulf Acute Heart Failure Registry. Angiology 2016; 68: 584-591.

15. Cuffe MS, Califf RM, Adams KF Jr, Benza R, Bourge R, Colucci WS, et al. Short-term intravenous milrinone for acute exacerbation of chronic heart failure: A randomized controlled trial. JAMA 2002; 287: 1541-1547.

16. Abraham WT, Adams KF, Fonarow GC, Costanzo MR, Berkowitz RL, LeJemtel TH, et al. In-hospital mortality in patients with acute decompensated heart failure requiring intravenous vasoactive medications: An analysis from the Acute Decompensated Heart Failure National Registry (ADHERE). $J$ Am Coll Cardiol 2005; 46: 57-64.

17. Levy WC, Mozaffarian D, Linker DT, Sutradhar SC, Anker SD, Cropp AB, et al. The Seattle heart failure model: Prediction of survival in heart failure. Circulation 2006; 113: 1424-1433.

18. Kajimoto K, Sato N, Takano T. Association of age and baseline systolic blood pressure with outcomes in patients hospitalized for acute heart failure syndromes. Int J Cardiol 2015; 191: 100-106.

19. Okuda N, Stamler J, Brown IJ, Ueshima H, Miura K, Okayama $\mathrm{A}$, et al. Individual efforts to reduce salt intake in China, Japan, UK, USA: What did people achieve? The INTERMAP population study. J Hypertens 2014; 32: 2385-2392.

20. Volpe M, Tritto C, DeLuca N, Rubattu S, Rao MA, Lamenza $\mathrm{F}$, et al. Abnormalities of sodium handling and of cardiovascular adaptations during high salt diet in patients with mild heart failure. Circulation 1993; 88: 1620-1627.

21. Kawaguchi M, Hay I, Fetics B, Kass DA. Combined ventricular systolic and arterial stiffening in patients with heart failure and preserved ejection fraction. Circulation 2003; 107: 714-720.

22. Cotter G, Metzkor E, Kaluski E, Faigenberg Z, Miller R, Simovitz A, et al. Randomised trial of high-dose isosorbide dinitrate plus low-dose furosemide versus high-dose furosemide plus low-dose isosorbide dinitrate in severe pulmonary oedema. Lancet 1998; 351: 389-393.

23. Doughty RN, Whalley GA, Gamble G, MacMahon S, Sharpe N. Left ventricular remodeling with carvedilol in patients with congestive heart failure due to ischemic heart disease. $\mathrm{J} \mathrm{Am} \mathrm{Coll}$ Cardiol 1997; 29: 1060-1066.

24. Maurer MS, Burkhoff D, Fried LP, Gottdiener J, King DL, Kitzman DW. Ventricular structure and function in hypertensive participants with heart failure and a normal ejection fraction: The Cardiovascular Health Study. J Am Coll Cardiol 2007; 49: 972-981.

25. Hanna EB, Glancy DL, Helmcke F. Left ventricular diastolic size in patients with normal ejection fraction and elevated left filling pressures. Echocardiography 2010; 27: 501-504.

26. Shah AM, Shah SJ, Anand IS, Sweitzer NK, O'Meara E, Heitner JF, et al. Cardiac structure and function in heart failure with preserved ejection fraction: Baseline findings from the echocardiographic study of the Treatment Of Preserved Cardiac Function Heart Failure with an Aldosterone Antagonist (TOPCAT) Trial. Circ Heart Fail 2014; 7: 740-751.

27. De Marco M, Gerdts E, Mancusi C, Roman MJ, Lønnebakken MT, Lee ET, et al. Influence of left ventricular stroke volume on incident heart failure in a population with preserved ejection fraction (from the Strong Heart Study). Am J Cardiol 2017; 119: $1047-1052$.

28. Mohammed SF, Borlaug BA, McNulty S, Lewis GD, Lin G, Zakeri R, et al. Resting ventricular-vascular function and exercise capacity in heart failure with preserved ejection fraction: A RELAX Trial ancillary study. Circ Heart Fail 2014; 7: 580-589.

29. Cohn JN, Ferrari R, Sharpe N. Cardiac remodeling: Concepts and clinical implications: A consensus paper from an international forum on cardiac remodeling. J Am Coll Cardiol 2000; 35: $569-582$.

30. Ather S, Chan W, Bozkurt B, Aguilar D, Ramasubbu K, Zachariah AA, et al. Impact of non-cardiac comorbidities on morbidity and mortality in a predominantly male population with heart failure and preserved versus reduced ejection fraction. J Am Coll Cardiol 2012; 59: 998-1005.

31. Wolsk E, Claggett B, Køber L, Pocock S, Yusuf S, Swedberg K, et al. Contribution of cardiac and extra-cardiac disease burden to risk of cardiovascular outcomes varies by ejection fraction in heart failure. Eur J Heart Fail 2017; 20: 504-510.

\section{Supplementary Files}

Please find supplementary file(s);

http://dx.doi.org/10.1253/circrep.CR-18-0013 